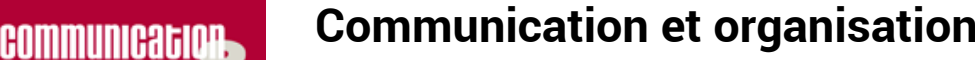

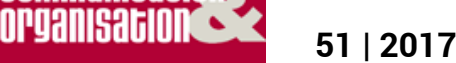

Les nouvelles cultures de l'information dans les organisations

\section{Les nouvelles cultures de l'information dans les organisations}

The new cultures of information within organizations

Vincent Liquète, Ivone de Lourdes Oliveira et Angela Salgueiro Marques

\section{OpenEdition}

\section{Journals}

Édition électronique

URL : http://journals.openedition.org/communicationorganisation/5492

DOI : 10.4000/communicationorganisation.5492

ISBN : 979-10-300-0146-4

ISSN : 1775-3546

Éditeur

Presses universitaires de Bordeaux

Édition imprimée

Date de publication : 1 juin 2017

Pagination : 5-11

ISBN : 979-10-300-0162-4

ISSN : 1168-5549

Référence électronique

Vincent Liquète, Ivone de Lourdes Oliveira et Angela Salgueiro Marques, «Les nouvelles cultures de l'information dans les organisations », Communication et organisation [En ligne], 51 | 2017, mis en ligne le 01 juin 2017, consulté le 19 novembre 2020. URL : http://journals.openedition.org/

communicationorganisation/5492 ; DOI : https://doi.org/10.4000/communicationorganisation.5492 


\title{
Les nouvelles cultures de l'information dans les organisations
}

\author{
Vincent Liquète ${ }^{1}$, Ivone de Lourdes Oliveira ${ }^{2}$ et \\ Angela Solgueiro Marques ${ }^{3}$
}

Issu des pays à économie forte, le concept de «culture de l'information » au sens anthropologique (Jenkins 2013) et communicationnel (Liquète 2014) du terme, désigne un ensemble de pratiques informationnelles articulant des connaissances issues de la culture académique traditionnelle (école, université, entreprise) avec celles plus intuitives et moins formalisées liées à l'information, aux documents et plus largement aux médias de masse. La culture de l'information repose ainsi sur des pratiques orales, de lecture, d'écriture et de mise en partage et en communication, dans des contextes à plus ou moins fortes prescriptions, qui sont observables dans des environnements académiques d'apprentissage, professionnels, ou culturels. De plus, le rapport culturel à l'information envisage une articulation entre des pratiques formelles et moins formelles, issues du monde social ou plus personnelles voire intimes. Originellement, ce concept de « culture de l'information » se déclinait à la forme singulière ; ces dernières années, la forme plurielle a pris le pas, «les cultures de l'information " pour montrer notamment une diversité de pratiques et une multiplicité de représentations selon les acteurs et/ou les environnements considérés.

Ainsi, face à cet émiettement des activités informationnelles, nombre de chercheurs plaident pour la forme plurielle (Liquète 2014) ; nous considérons ce caractère pluriel dans ce numéro. Les approches par les cultures de l'information plaident pour un dépassement des seules pratiques performatives technologiques de la recherche d'information ; ainsi, dès les années 1950, Gilbert Simondon (2012) considérait la technique comme un objet opératoire à penser avec l'homme et non pas contre lui. Comme le

1 Vincent Liquète est Professeur en SIC, Université de Bordeaux-France, IMS CNRS UMR 5218, Equipe RUDII.

2 Ivone de Lourdes Oliveira est Professeure à l'Université pontificale catholique du Minas Gerais, PUC-Minas (Belo Horizonte).

3 Angela Salgueiro Marques est Professeure du Programme de Post-Graduation à l'Université Fédérale de Minas Gerais (Belo Horizonte). 
souligne Alexandre Serres (2014), la culture de l'information s'est construire autour d'un périmètre large considérant des comportements personnels, des gestes cognitifs, des valeurs, des pratiques, des savoirs théoriques, des savoirfaire sur l'information (Baltz 1998).

Ces vingt dernières années, les travaux autour de la dimension culturelle des pratiques d'information se sont principalement orientés autour des secteurs de l'école, des universités et des bibliothèques (Le Deuff 2009), et vers des problématiques liées à la question de l'acquisition de compétences et des connaissances. L'approche orientée usage(r)s (Le Coadic 1997) visait une analyse compréhensive des usages de l'information dans le secteur des organisations et des entreprises mais restait avant tout focalisée sur le repérage et l'analyse compréhensive des pratiques d'information liées à des techniques, des dispositifs, ou des ressources. Plus récemment, les approches de la théorie du management de l'information considèrent qu'une gestion rationnelle des gisements, des accès et des contenus d'information, notamment professionnelle permet de renforcer l'efficacité de l'organisation dans son secteur d'activités ou de rayonnement ; or, comme le souligne Brigitte Guyot (2012), il est difficile de savoir si la gestion de l'information a pu changer une situation dans une organisation si ce n'est en interrogeant celle-ci par le biais de ses salariés et managers. Dans des organisations plus modestes, les responsables de micro-entreprises, d'associations, de clusters, par exemple, n'ont effectivement pas toujours recours aux techniques d'étude et d'évaluation du besoin d'information et encore moins à l'analyse des conditions de création, d'échange et de partage de celle-ci au sein de ses murs et de ses missions.

Au gré de la préparation de ce numéro, nous avons également constaté que les études et recherches sur les cultures de l'information ont finalement peu pris en considération les dimensions communicationnelles. En effet, au-delà des seules formes d'incommunication, force est de constater que les cultures de l'information méritent d'être interrogées au prisme des théories et des cribles des théories de la communication : $y$-a-t-il des dispositifs, des situations, des contextes info-communicationnels favorables ou obstacles aux cultures de l'information en situation?

Nous considèrerons dans ce numéro, sans exception, la plus large acception de la notion d'organisation, de la micro-entreprise aux grandes organisations, dans des secteurs d'activités variés et variables, tout en considérant des périmètres géographiques variés avec cette approche bipolaire France - Brésil, cultures sud-américaine et européenne. Nous tenterons ainsi, par les diverses contributions retenues, de comprendre les tendances sociales et professionnelles partagées qui semblent se dessiner face à l'émiettement des pratiques d'information, de voir dans quelles mesures l'évolution des systèmes d'information, de documentation et de communication, qui tendent à se rapprocher et à inter-opérer, transforment les rapports à l'information et à la communication à tel point quelle permet l'émergence de cultures 
ou de micro-cultures, d'identifier et de caractériser les formes d'affiliation en jeu entre les individus afin de transmettre une part de savoir-faire et de compétences par l'activité, enfin, de considérer un rapprochement de plus en plus identifiable entre les pratiques numériques sociales et les démarches infocommunicationnelles attendues et soutenues au sein même des organisations. Ainsi, comme le suggère A. Perrin (2008), avec le développement $\mathrm{d}$ ' « une forte culture de partage d'information ", la circulation de l'information est d'autant plus importante que celle-ci n'est alors plus considérée uniquement comme une source de pouvoir et de contrôle de l'autre, même si elle perdure sous diverses formes ; la circulation voire le partage de l'information devient le ciment de formes de cohésion du groupe et de construction collective négociée de la connaissance, où le perspective communicationnelle est centrale. Penser ainsi les cultures de l'information dans les organisations revient à mettre la focale sur la culture organisationnelle basée sur la collaboration et la confiance favorisant de surcroît le partage des connaissances et leur diffusion. Sans omettre pour autant, des formes de résistance, de contournement voire de détournement plus ou moins intentionnels. G. Le Cardinal rappelle que « $\mathrm{La}$ confiance est ce qui permet la prise de risques dans les relations humaines, c'est le non-dit qui lie les associés face à l'explicite du contrat, c'est la connivence opposée au contrôle tatillon, c'est l'explication de la dynamique des relations humaines en évolution incessante que ce soit vers la coopération confiante ou le conflit ouvert».

Ce numéro cherche à convoquer des approches complémentaires, l'une centrée sur la délimitation épistémologique et théorique du concept même de culture(s) de l'information, en lien avec la communication des organisations ; une autre approche, vise à questionner la sélection, l'appropriation et l'utilisation de l'information dans les organisations, selon des perspectives communicationnelles; une troisième cherche à mettre en lumière des recherches issues de secteurs d'activité variés (entreprises, monde scolaire, monde universitaire, associations, espaces culturels ou bibliothéconomiques, ...) révélant des cultures de l'information en activité et en situation sous des formes multiples; enfin, ce numéro vise un aspect prospectif montrant des perspectives qui semblent progressivement se dessiner dans certains domaines d'activités des organisations.

Ainsi, nous avons retenu sept contributions dans ce numéro :

Anne Lehmans dans «Données ouvertes et redéfinition de la culture de l'information dans les organisations : vers une culture de la donnée » argumente que la mise en place des politiques publiques ne va pas nécessairement de pair avec leur mise en œuvre dans les organisations, parce que l'ouverture des données représente un changement profond, du point de vue du management de l'information notamment. Cette ouverture a pour objectif affiché et utopique la transparence, la participation citoyenne et la 
modernisation de l'action publique. Néanmoins, l'émancipation citoyenne requiert la prise en compte des dimensions pragmatiques de la politique et la problématisation d'une gouvernance biopolitique qui rend difficile la médiation des données ouvertes en, par exemple, l'éducation. L'article discute comment les collectivités territoriales en région aquitaine qui ont mis en place une politique d'ouverture et médiation des données. Le projet sur la médiation des données pour l'éducation a réussi a montrer les conditions de construction d'une véritable culture de l'information intégrant la donnée chez les enseignants qui pourraient en faire un usage pédagogique. Il s'agit de faire émerger une culture partagée des données, enjeu de leur réappropriation effective par la société civile et dans les entreprises.

Smail Khainnar dans « La culture de l'information en milieu universitaire : Application à une démarche collaborative de création d'une "start-up communicante " présente une étude appliquée à un contexte universitaire afin d'enquêter la façon dont la mise en place de la notion de culture de l'information peut transformer une situation pédagogique. Une vingtaine d'étudiants inscrits en première année du master IN2C (Ingénierie Numérique Collaborative pour la Construction) ouvert cette année universitaire 2016/2017 à l'UVHC (Université de Valenciennes et du Hainaut Cambrésis) ont participé à la recherche. En essayant de produire une délimitation conceptuelle de la culture de l'information dans sa déclinaison pédagogique, Khainnar argumente que, dans le cadre du module " communication interacteurs ", prévu dans la maquette pédagogique de la formation IN2C et ayant un volume horaire de $12 \mathrm{~h} \mathrm{CM}$ et $6 \mathrm{~h} \mathrm{TD}$, il fallait interroger les pratiques informationnelles (recherche, trouvabilité, partage, exploitation, etc.) initiées, conduites et gérées par des étudiants travaillant en groupe pour la création d'une « start-up communicante ». À travers une approche qualitative il analyse les pratiques informationnelles issues de cette expérimentation, pour montrer les imbrications entre quatre dimensions de la culture de l'information : le savoir-faire, le savoir, la réflexion critique et la réflexivité (l'autoréflexion).

Jessica de Bideran et Mélanie Bourdaa dans «Quand les experts du patrimoine s'emparent du transmédia storytelling. L'exemple de Montaigne Superstar, une stratégie inachevée » montrent comment s'élaborent progressivement des stratégies informationnelles et communicationnelles qui s'inscrivent dans une logique de convergence culturelle et technologique. Les auteurs veulent réfléchir à propos de l'intégration des cultures numériques au sein d'une organisation où la gestion de l'information, notamment documentaire, est essentielle, la bibliothèque. Elles soutiennent que le transmédia storytelling s'applique parfaitement aux projets portés par l'industrie du divertissement hollywoodienne qui dispose de moyens financiers considérables comme d'importantes communautés de fans susceptibles de 
servir de caisses de résonance, celle-ci subit quelques distorsions lorsqu'on l'applique à l'univers du patrimoine et de ses acteurs. L'analyse du Montaigne Superstar nous donne à voir les évolutions en cours au sein de la bibliothèque municipale de Bordeaux. Car c'est finalement en termes de structuration que cette expérimentation a été la plus pertinente ; ce projet et la montée en compétences qu'il a imposée à l'équipe concernée a ainsi permis d'affirmer avec force la nouvelle politique scientifique de l'établissement fermement orientée désormais vers la médiation numérique et l'univers ludique.

Aurélia Dumas, Fabienne Martin-Juchat et Julien Pierre dans « Les cadres et le numérique : cultures informationnelles et sentiment d'efficacité » analysent la corrélation entre sentiment d'efficacité au travail et cultures de l'information. Par des entretiens approfondis, elles ont cherché à saisir les bricolages et aptitudes développés au travail, mais rares sont les " tribus " professionnelles qui partagent collectivement un savoir-faire des outils intégrant des logiciels de gestion de l'information. Il y a ainsi un risque d'épuisement des ressources cognitivo-affectives et d'improductivité dans l'activité de travail. Les auteurs affirment que le sentiment d'efficacité au travail est corrélatif à la maîtrise affective des outils. Elles ont observé l'émergence d'un «travail émotionnel » se faisant aptitudes émotionnelles, dans la manière de gérer les ressources numériques, en fonction des contextes et des cultures organisationnels associés : ces aptitudes, dont fait partie la maîtrise des émotions, conditionnent la capacité à gérer les flux informationnels. A ce titre, les réseaux sociaux grand public sont très couramment perçus par les salariés interrogés comme sources de dispersion et de perte de temps, leur demandant de développer de fortes aptitudes à l'autocontrôle. Le sentiment d'efficacité au travail se trouve donc en partie lié aux capacités à gérer les flux et, plus spécifiquement, à les réguler dans un contexte de porosité voire de disparition des frontières entre vie professionnelle et vie privée.

Anne Cordier et Angèle Stalder dans " Modes d'appréhension et de dissémination des cultures de l'information dans l'École : les conditions de la reliance " visent à penser le lien entre la considération des cultures de l'information dans l'organisation - scolaire et professionnelle, particulièrement - et la reliance. C'est le processus de reliance qui intègre des connaissances et des cultures, de même que les individus, fédérés par le souci de mener à bien une action collective. Pour elles l'École est une organisation apprenante, comparable à une entreprise " innovante ", amenée à pointer les pratiques et dispositions qu'elle peut mettre en œuvre pour évoluer avec son écosystème informationnel et tenter de repérer les dimensions organisationnelles de l'apprentissage. Les auteurs entreprennent l'investigation des dispositifs info-communicationnels engageant les acteurs dans une démarche de co-construction de savoirs à travers la compréhension et l'appropriation de notions et compétences 
informationnels. Les analyses visent à identifier les conditions de la reliance e à montrer comment l'organisation apprenante qu'est l'École peut favoriser de manière effective la culture des liens entre des univers différents, dépassant les cloisonnements disciplinaires, pour atteindre une véritable dissémination des cultures de l'information. Penser les conditions de la reliance pour les cultures de l'information dans l'organisation scolaire, selon elles, c'est donc éviter l'aspect mosaïque trop souvent revêtu par les analyses des pratiques informationnelles, réalisées selon un système de couches (d'individus isolés selon les statuts, par exemple, ou encore de types d'activités observées), et tenter de saisir les niches de création du sens individuel et social de l'activité.

Tamires Ferreira Coêlho et Marcelo Rodrigo da Silva dans « La mort, l'humour et les stratégies de communication numérique " proposent un approche de l'humour à partir de la construction des stratégies de communication, et traiter la thématique de la mort par un modèle d'organisation classiquement peu étudié : le cimetière. Une analyse des tactiques d'annonces véhiculées sur la page Facebook du cimetière «Jardim da Ressureição - Cemitério Parque», situé à Teresina/PI montre que le contenu humoristique diffusé par les pages organisationnelles établit des liens entre les pratiques numériques et la «culture de l'information» dans ces institutions. Le processus de médiatisation et la nouvelle architecture communicationnelle du réseau numérique mettent en lumière de nouveaux protocoles et formes de circulation des sens qui, à leur tour, ne se restreignent pas aux savoirs produits formellement dans le domaine de l'institution. La production de contenus pour internet est imprégnée de savoirs non traditionnels, basée sur le langage oral, sur les écritures et les réécritures, sur l'association entre le partage et la performance qui déplace les processus communicatifs au-delà des pôles rigides de production et de réception.

Rosângela Florczak et Cleusa Maria de Andrade Scroferneker dans «De la perspective informationnelle aux (im)possibilités relationnelles de la communication dans le contexte des organisations ", soutiennent que les processus communicationnels qui construisent les organisations sont marqués par des conflits d'intérêts et des logiques distinctes nuancés par les relations de pouvoir. Cette perspective rompre avec une vision réductionniste/linéaire/ prescriptive de la communication organisationnelle pour signaler d'autres possibilités révélant en partie l'épuisement du paradigme transmissif. Selon elles, étudier la communication dans le contexte organisationnel c'est analyser la relation entre sujets interlocuteurs qui négocient du sens dans l'interaction entendue comme flux, comme une construction négociée, où la relation se (re) définit en permanence. Cet approche nous permet de redéfinir les concepts et requiert l'inclusion de la communication stratégique, fruit d'une pensée complexe, dans les cadres d'interpellation réciproque qui impliquent non 
seulement de parler et de diffuser, mais également d'écouter et de dialoguer, de rechercher l'articulation plutôt que la persuasion, la connectivité plutôt que la diffusion, le partage et l'harmonisation des perceptions et des valeurs plutôt que leur imposition. Étant donné que la communication est le lieu de rencontre et de construction de significations et de sens partagés, il faut considérer que les nouvelles cultures de l'information dans les organisations donnent origine à des nouvelles optiques paradigmatiques pour délimiter le champ, l'objet et les caractéristiques qui représentent le phénomène communicationnel/ organisationnel, et ce sans nécessairement oublier le dissensus et le rôle de la stratégie. 